\title{
The Relationship Between Patients' Anthropometric Characteristics and Depth of Spinal Needle Insertion
}

\author{
Mohammad Reza Razavizadeh, ${ }^{1}$ Mohammad Reza Fazel, ${ }^{1, *}$ Mahdi Mosavi, ${ }^{2}$ and Mojtaba \\ Sehat ${ }^{3}$ \\ ${ }^{1}$ Trauma Research Center, Kashan University of Medical Sciences, Kashan, Iran \\ ${ }^{2}$ Department of Anesthesiology, Kashan University of Medical Sciences, Kashan, Iran \\ ${ }^{3}$ Department of Community Medicine, Kashan University of Medical Sciences, Kashan, Iran \\ ${ }^{*}$ Corresponding author: Mohammad Reza Fazel, Trauma Research Center, Kashan University of Medical Sciences, Kashan, Iran. Tel: +98-9132760380, Fax: +98-3615342025, E- \\ mail: drmfazel @yahoo.com
}

Received 2014 October 29; Revised 2015 December 4; Accepted 2016 January 4.

\begin{abstract}
Background: Many surgeries are performed under spinal anesthesia. Inexperienced practitioners may find it difficult to obtain subarachnoid access.

Objectives: This study aimed to examine the relationship between patients' anthropometric characteristics and depth of spinal needle insertion to the subarachnoid cavity.

Patients and Methods: 385 patients with ASA class I - II, aged 18 - 65 years and undergoing elective surgery of the lower abdomen and extremities under spinal anesthesia, were selected for this cross-sectional study. The patients' demographic characteristics, body mass index (BMI), and anthropometric characteristics (height, weight, waist circumference, and arm circumference) were recorded. Linear regression and t-student tests were used to study the relationship between anthropometric characteristics and BMI, and depth of needle insertion.

Results: Of the 385 patients studied, 88 were female and 297 were male. There was a strong correlation between the depth of needle insertion and BMI $(24.9 \pm 3.9)$, and between depth and weight/height ratio $(r=0.95$ and $r=0.92$, respectively). There was no significant correlation between depth of needle insertion and weight, height, gender, or arm circumference, when considered separately. The statistical predicting models showed that the following relationship was observed between the needle depth and the weight/height ratio: A: needle depth $=0.69+(10.1 \times$ weight $/$ height $)$; : needle depth $=0.56+(0.18 \times \mathrm{BMI})$.

Conclusions: The results of this study show that there is a strong relationship between depth of needle insertion and BMI, and between depth and the weight/height ratio; appropriate depths can be determined according to the equations obtained.
\end{abstract}

Keywords: Needles, Spinal Puncture, Distance Perception

\section{Background}

Many lower limb and lower abdominal surgeries are performed under spinal anesthesia. Subarachnoid access may be difficult to perform for inexperienced practitioners, or when patients have either an unusually large or small body habitus. Prior knowledge of how far the needle needs to be inserted to reach the intrathecal space may reduce the potential complications of spinal anesthesia (1). Multiple attempts to place the needle into the subarachnoid space not only cause discomfort and dissatisfaction for the patient, they can also increase the likelihood of post-punctural headaches (2), neurological damage, (3), and epidural hematoma (4).

\section{Objectives}

Through this study, we would like to design a practical measure for estimating the depth of needle insertion, to attain successful spinal anesthesia based on the patient's anthropometric characteristics.

\section{Patients and Methods}

After approval by the ethics committee of the university, and the obtaining of written informed consent from all subjects 385 patients with ASA class I - II, aged 18 - 65 and undergoing elective surgery on their lower abdomen and limbs under spinal anesthesia, who were admitted to Shahid Beheshti hospital of Kashan University of Medical Sciences (KAUMS) during 2012, were enrolled in this crosssectional study. Before surgery, height, weight, gender, body mass index (BMI), waist circumference (WC), the line that passes across the upper iliac crest's edge in a sitting position, and the arm circumference (exactly $10 \mathrm{~cm}$ above the cubital fossa) were measured. In addition, the

Copyright ( 2016, Iranian Society of Regional Anesthesia and Pain Medicine(ISRAPM). This is an open-access article distributed under the terms of the Creative Commons Attribution-NonCommercial 4.0 International License (http://creativecommons.org/licenses/by-nc/4.0/) which permits copy and redistribute the material just in noncommercial usages, provided the original work is properly cited. 
spinal anesthesia method was explained to the patients, and their consent was obtained for performing the block. Pregnant women were excluded from the study. In the operating room, the anesthesiologist (who had experience of at least 500 cases of spinal block), after preparing the patient, performed the spinal anesthesia using a $25 \mathrm{G}$ cutting (Qinke) needle through the midline at L4 - 5 and L5 - S1 level (using Tuffier's line) in the sitting position. After the medication was injected, the insertion point was marked on the needle, which was then removed. The depth to which the needle had entered the patient's body was then carefully measured with a caliper and recorded. Patients with a skin infection at the site of the block, who were unable to maintain sitting position without assistance, who exhibited kyphosis, lordosis, or scoliosis, or who had a failed block were excluded from the study. The main statistical tests used were linear regression and tstudent test. A Pearson correlation coefficient of $r>0.7$ indicated a strong relationship between dependent and independent parameters; the significant level was set at $\mathrm{P}<0.05$. SPSS software, version 13.0 (SPSS Inc., Chicago, Illinois, USA) was used for data analysis.

\section{Results}

Demographic details of the 385 patients who participated in the study are presented in Table 1 . Based on the regression analysis between the needle depth and study variables, the following results were obtained:

Table 1. Demographic and Procedural Details of Patients Undergoing Spinal Anesthesia

\begin{tabular}{|lc}
\hline Demographic/Procedural Data & Values \\
\hline Age, $\mathbf{y}$ & $37.25 \pm 13.37$ \\
\hline Gender & \\
\hline Male & 297 \\
\hline Female & 88 \\
\hline Type of surgery & \\
\hline Lower abdominal & 105 \\
\hline Urology & 79 \\
\hline Gynecology & 46 \\
\hline Orthopedic & 155 \\
\hline Weight, kg & $72.7 \pm 12.1(37.0-107)$ \\
\hline Height, cm & $170.7 \pm 7.4(152.0-190)$ \\
\hline BMI, kg/m ${ }^{2}$ & $24.9 \pm 3.9(15.4-40.1)$ \\
\hline Arm circumference, cm & $27.9 \pm 4.9(15.0-87.0)$ \\
\hline Waist circumference, cm & $84.7 \pm 12.4(28.0-133.0)$ \\
\hline Spinal needle Depth ,cm & $3.3-7.8$ \\
\hline Spinal needle Insertion Space, & \\
\hline No. $\%$ ) & \\
\hline L4/L5 & $331(86)$ \\
\hline L5/S1 & $54(14)$ \\
\hline Ab & \\
\hline
\end{tabular}

Abbreviation: BMI, body mass index.

\subsection{Depth of Needle Insertion and Weight Correlation}

The linear correlation between depth of needle insertion and weight was significant; $0.66 \%$ variation in depth of needle insertion will be interpretable by this model. Pearson correlation coefficient $(r)=0.817 ; \mathrm{P}<0.001$.

\subsection{Depth of Needle Insertion and Height Correlation}

The correlation between depth of needle insertion variables and patients' height was inverse and insignificant. This model can only explain about $9 \%$ of depth of needle insertion variables $(\mathrm{P}=0.091, \mathrm{r}=0.107)$.

\subsection{Depth of Needle Insertion and Body Mass Index Correlation}

There was a significant correlation between depth of needle insertion and BMI. Based on the $\mathrm{R}^{2}$ calculation in the regression model, nearly $90 \%$ of depth of needle insertion variables can be interpreted by this model. As shown in Figure 1, this correlation was very strong and significant $(\mathrm{P}<0.001, \mathrm{r}=0.95)$.

\subsection{Arm Circumference and Depth of Needle Inser- tion Correlation}

The correlation between these parameters was significant but weak, and only $40 \%$ of depth of needle insertion variables were predictable using this model $(\mathrm{P}<0.001, \mathrm{r}$ $=0.630$ ).

\subsection{The Waist Circumferenc (WC) and Depth of Needle Insertion Correlation}

The correlation between the WC and depth of needle insertion was significant; however, according to the regression model, only $43 \%$ of depth of needle insertion variables were predictable using this model $(\mathrm{P}<0.001, \mathrm{r}$ $=0.657$ ) (Figure 2 ).

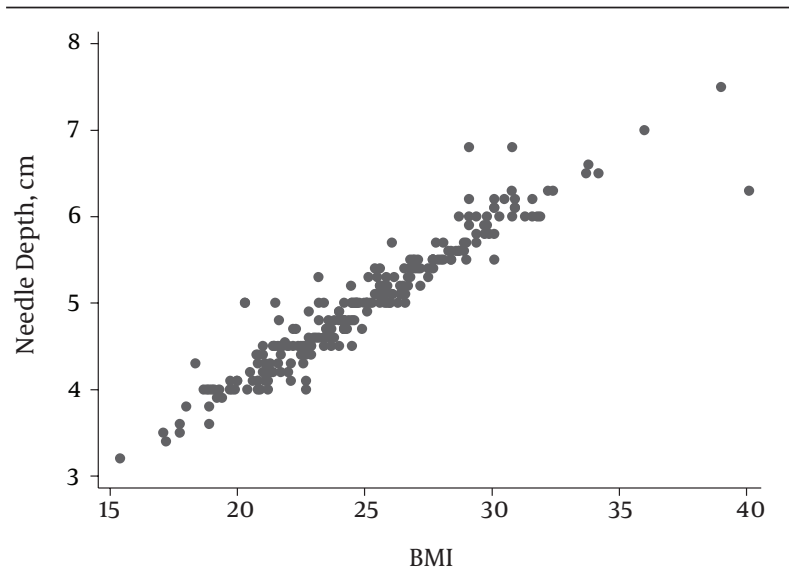

Figure 1. Correlation Between Depth of Needle Insertion and Body Mass Index in Patients 


\subsection{Correlation Between age and Depth of Needle Insertion}

In this study, age was considered as an independent parameter when determining the depth of needle insertion. There was no significant correlation between age and depth of needle insertion $(r=0.12, P=0.6)$. Considering that most of the patients were adults over 20 years of age, a significant change in the depth of needle insertion is not expected in this age group.

\subsection{Correlation Between Weight/Height Ratio and Depth of Needle Insertion}

The weight/height ratio is an index used to predict depth of needle insertion. In this model, the Pearson correlation coefficient between these parameters is significant $(\mathrm{r}=0.917, \mathrm{P}<0.001) .84 \%$ of needle changes are predictable using this model (Figure 3 ). The best model for predicting depth of needle insertion, according to the weight/height ratio with the least residual, is:

Needle depth $=0.69+(10.1 \times \mathrm{W} / \mathrm{H})$

\subsection{Needle Depth Correlation With Gender and Study Variables}

In terms of sex (male and female), there was no significant correlation between depth of needle insertion and arm circumference $(P=0.187)$, weight $(P=0.665)$, height $(\mathrm{P}=0.11)$, BMI $(\mathrm{P}=0.54)$, and weight $/$ height ratio $(\mathrm{P}=$ $0.874)$. The WC in women had a higher correlation with depth of needle insertion, and the difference between males and females was significant $(\mathrm{P}=0.046)$ (Table 2). The best statistical model for predicting the spinal depth of needle insertion was in the form of the two equations for the depth of needle insertion correlations with weight/height ratio (graph A) and BMI (graph B):

A: Needle depth $=0.69+(10.1 \times \mathrm{W} / \mathrm{H})$

B: Needle depth $=0.56+(0.18 \times \mathrm{BMI})$

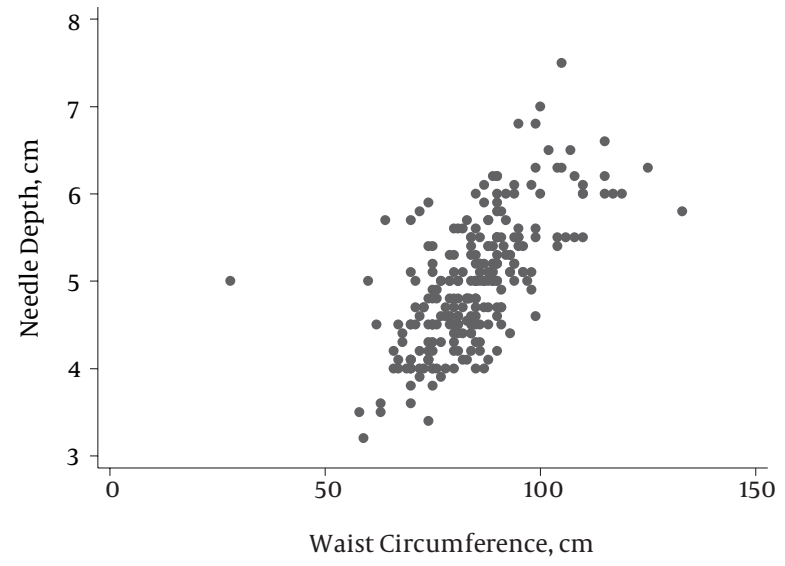

Figure 2. Correlation Between Depth of Needle Insertion and Waist Circumference in Patients

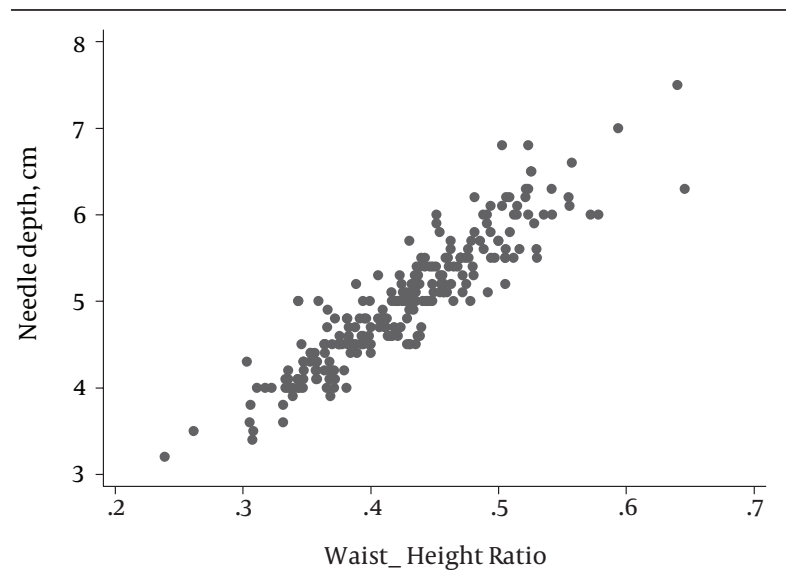

Figure 3. Depth of Needle Insertion Correlation With Weight/Height Ratio

Table 2. Correlation Between Needle Depth and Anthropometric Indices

\begin{tabular}{|c|c|c|c|c|c|c|c|c|c|c|}
\hline \multirow[t]{2}{*}{ Parameter } & \multicolumn{3}{|c|}{ Male } & \multicolumn{3}{|c|}{ Female } & \multicolumn{3}{|c|}{ Total } & \multirow[t]{2}{*}{ P Value } \\
\hline & $\mathbf{r}$ & PValue & $\mathbf{R}^{2}$ & $\mathbf{r}$ & P Value & $\mathbf{R}^{2}$ & $\mathbf{r}$ & P Value & $\mathbf{R}^{2}$ & \\
\hline Weight & 0.82 & $<0.001$ & 0.67 & 0.801 & $<0.001$ & 0.64 & 0.817 & $<0.001$ & 0.66 & 0.67 \\
\hline Height & 0.058 & 0.422 & 0.003 & 0.227 & 0.037 & 0.08 & 0.107 & 0.09 & 0.01 & 0.11 \\
\hline BMI & 0.948 & $<0.001$ & 0.9 & 0.963 & $<0.001$ & 0.93 & 0.952 & $<0.001$ & 0.9 & 0.55 \\
\hline AC & 0.668 & $<0.001$ & 0.44 & 0.48 & $<0.001$ & 0.23 & 0.63 & $<0.001$ & 0.4 & 0.19 \\
\hline WC & 0.654 & $<0.001$ & 0.42 & 0.68 & $<0.001$ & 0.46 & 0.657 & $<0.001$ & 0.43 & 0.04 \\
\hline Age & 0.099 & 0.171 & 0.01 & 0.188 & 0.162 & 0.03 & 0.12 & 0.057 & 0.01 & 0.13 \\
\hline $\mathbf{W} / \mathbf{H}$ & 0.915 & $<0.001$ & 0.84 & 0.919 & $<0.001$ & 0.84 & 0.917 & $<0.001$ & 0.84 & 0.87 \\
\hline
\end{tabular}

Abbreviations: AC, arm circumference; BMI, body mass index; WC, waist circumference; W/H, weight/height. 


\section{Discussion}

Although spinal anesthesia is usually straightforward, it can be technically challenging with patients who are of unusually large or small body habitus. In addition, less experienced practitioners have more difficulty performing it. A formula that can predict the depth of needle insertion in overly large or small patients would increase accuracy in the performance of spinal block, especially for clinicians with less experience; it would also enhance patients' comfort and satisfaction, as well as expediting their procedure time. In a study by Craig et al. (5) on patients aged 0.01 - 16 years, conducted to predict the lumber puncture (LP) depth of needle insertion, the relationship between depth of needle insertion and the height, weight, and age of the patients was analyzed. A linear relationship was found between the child's height and the depth of needle insertion; as a result, the mean depth of insertion could be determined by $0.03 \times$ height $(\mathrm{cm})$. In the present study, conducted on patients aged 18 - 65 years, the depth of needle insertion had an inverse relationship with height, which was not strongly significant. In Craig et al. the regression constant was ignored, which meant that other influential factors in the study were not considered. In addition, the age groups in the two studies are not compatible. The present study was conducted on patients that had completed their development in terms of height, while the study group investigated by Craig et al. consisted of children in their formative years. Growth at this age depends on several factors such as nutrition, as well as hugely diverse natural characteristics, which can adversely affect the results and their generalization. In another study by Chong et al. (6) on 279 Chinese patients aged six months to 15 years, multiple regression tests showed a strong relationship between the lumbar puncture depth of needle insertion and the weight/height ratio. By using a predictive regression model, the ideal depth of needle insertion $(\mathrm{cm})$ was determined as 10 [weight $(\mathrm{kg}) /$ height $(\mathrm{cm})]+$ 0.93 , with a regression coefficient of $r=0.77$. In the present study, the depth of needle insertion's relationship with the weight/height ratio was defined by the equation 0.69 $+(10.1 \times$ weight $/$ height $)$. The difference in the coefficient of the weight/height ratio between these two studies could be due to ethnic differences, as well as the different age groups, which affects the anthropometric characteristics of patients. Chong et al's study was conducted on Chinese patients, who are known to be of generally smaller stature. In addition, the study group included children who were still in their formative years and not yet fully developed, compared to adults whose height stays almost constant after puberty. In a study by Arzola et al. (7), the relationship between patients' anthropometric parameters and the skin to lumbar dural sac distance was investigated; it was shown that this distance had a significant correlation with patients' weight and BMI, which is in agreement with the results of the present study. In Arzola et al's study, a formula was not provided for predicting depth of needle insertion, but in the current study, the relationship between depth of needle insertion and BMI was expressed as depth of needle insertion $=0.56+(0.18 \times \mathrm{BMI})$, providing a means of prediction of the needle length. According to the regression model, in our study, the relationship between weight and BMI with the depth of needle insertion is stronger than that presented in Arzola et al's study. This may be due to the group they were investigating, which consisted of pregnant women who were delivering under epidural block (7). During pregnancy, the mother's weight and her lumbar lordosis undergo major changes, which could affect the accuracy of study results and generalization to other patients $(8,9)$. In addition, weight gain in pregnancy is not constant or equal in all women, as it differs by 7 - $14 \mathrm{~kg}$ (9). In a cohort study by Abe et al. (10) on 175 patients aged 25 days to 80 years, in patients undergoing CT scan for a variety of reasons, the LP depth in CT was measured and a formula for calculating LP needle depth was presented in the form of LP depth $=1+(17 \times \mathrm{W} / \mathrm{H})$. This study showed that the Abe formula was a more reliable predictor for estimating the required LP needle depth, in comparison with other published formulas $\left(\mathrm{R}^{2}=0.81\right)(10)$. Abe et al's study was in almost constant agreement with our paper in terms of the significant correlation between the LP needle depth and weight/height ratio. However, in the above mentioned study, the age range was very diverse, and due to the difference in speed of height and weight development in various age groups, the weight/height ratio may also have been affected. It seems that separate determination of this ratio for each age group could achieve a result closer to reality. Additionally, as stated by the authors, the weight/height ratio was derived from the patients' records, and may not necessarily have been reliable. In the present study, other anthropometric characteristics of patients were also measured (WC, biceps), and their correlation with the depth of needle insertion was analyzed and found to be not significant.

\section{Acknowledgments}

The authors would like to thank the deputy of research at Kashan University of Medical Sciences.

\section{Footnotes}

Authors' Contribution:Mohammad Reza Razavizadeh was in charge of ideas and the design and implementation of the study; Mohammad Reza Fazel supervised it; Mahdi Mosavi took responsibility for interpretation of the results, and Mojtaba Sehat performed the statistical analysis.

Funding/Support:This study was funded and supported by the deputy of research at Kashan University of Medical Sciences.

\section{References}

1. Ronald D. Miller MD. Brown DL. Spinal, epidural, and caudal anesthesia . 16 ed: Churchill Livingstone; 2005. pp.1653-8. 
2. Faitot V, Ourchane R, Dahmani S, Magheru M, Nebout S, Gomas $\mathrm{F}$, et al. An observational study of factors leading to difficulty in resident anaesthesiologists identifying the epidural space in obstetric patients. Int J Obstet Anesth. 2011;20(2):124-7. doi: 10.1016/j. ijoa.2010.11.008. [PubMed:21316214]

3. De Tommaso O, Caporuscio A, Tagariello V. Neurological complications following central neuraxial blocks: are there predictive factors? Eur J Anaesthesiol. 2002;19(10):705-16. [PubMed: 12463382]

4. Gottschalk A, Bischoff P, Lamszus K, Standl T. Epidural hematoma after spinal anesthesia in a patient with undiagnosed epidural lymphoma. Anesth Analg. 2004;98(4):1181-3. [PubMed: 15041622]

5. Craig F, Stroobant J, Winrow A, Davies H. Depth of insertion of a lumbar puncture needle. Arch Dis Child. 1997;77(5):450. [PubMed: 9487973]

6. Chong SY, Chong LA, Ariffin H. Accurate prediction of the needle depth required for successful lumbar puncture. Am J Emerg Med. 2010;28(5):603-6. doi: 10.1016/j.ajem.2009.02.006. [PubMed: 20579557]

7. Arzola C, Davies S, Rofaeel A, Carvalho JCA. Anthropometric variables and lumbar dural sac width using ultrasound. Can J Anaesth. 2006;53:26291.

8. Borg-Stein J, Dugan SA, Gruber J. Musculoskeletal aspects of pregnancy. Am J Phys Med Rehabil. 2005;84(3):180-92. [PubMed: 15725792]

9. Abrams B, Altman SL, Pickett KE. Pregnancy weight gain: still controversial. Am J Clin Nutr. 2000;71(5 Suppl):1233S-41S. [PubMed: 10799396]

10. Abe KK, Yamamoto LG, Itoman EM, Nakasone TA, Kanayama SK Lumbar puncture needle length determination. Am J Emerg Med. 2005;23(6):742-6. doi: 10.1016/j.ajem.2005.03.012. [PubMed: 16182981] 